\title{
INTRODUCTION TO HIGH-FREQUENCY RADAR: REALITY AND MYTH
}

\author{
By Jeffrey D. Paduan and Hans C. Graber
}

... we focus on the measurements of primary interest to coastal oceanographers ...
$\mathrm{T}$ HE CONCEPT OF USING high-frequency (HF) radio pulses to remotely probe the ocean surface has been around for decades. In this paper and the companion paper by Teague et al. (1997) we strive to introduce this technique to a broad oceanographic audience. Teague et al. (1997) provides the historical context plus an outline of different system configurations, whereas we focus on the measurements of primary interest to coastal oceanographers, i.e., maps of near-surface currents, wave heights, and wind direction. Another goal of this paper and, indeed, this entire issue is to present a realistic assessment of the state-of-the-art in $\mathrm{HF}$ radar techniques vis-à-vis coastal oceanography. When evaluating any new measurement technique, it is important to separate issues related to system design from fundamental limitations of the technique. The former are engineering shortcomings, which are subject to continuous improvement. The latter are real limitations in the use of the particular geophysical signal in the presence of realistic noise. Most of the "myths" about HF radar measurements, in our view, stem from the confusion of these two issues.

One common misconception about HF radar stems from the word "radar" itself. A more descriptive name would be HF "radio," as the HF portion of the electromagnetic spectrum is within the radio bands. Figure 1 shows a broad range of the electromagnetic spectrum, including the nomenclature commonly applied to different portions of the spectrum. The HF band, with frequencies of $\sim 3-30 \mathrm{MHz}$ and wavelengths of $\sim 10-100$ $m$. sits between the spectral bands used for television and (AM) radio transmissions. Often, the term radar is applied to instruments operating in the microwave portion of the spectrum, for which wavelengths are measured in millimeters or centimeters.

Throughout oceanography, many different instruments exploit many different portions of the

Jeffrey D. Paduan. Code OC/Pd, Naval Postgraduate School, Monterey, CA 93943, USA; Hans C. Graber, Rosenstiel School of Marine and Atmospheric Science. University of Miami, Miami, FL 33149-1049, USA electromagnetic spectrum. Figure 2 illustrates several of these remote sensing techniques used to extract information about the ocean surface. The figure is adapted from the review by Shearman (1981) and it contrasts space-borne systems, such as altimeters and scatterometers, which use microwave frequencies, with shore-based systems, which use a range of frequencies depending on the application. (Not shown are aircraft-borne systems, which also operate in the microwave band.) The figure also illustrates the different types of transmission paths, including true line-of-sight paths, "sky wave" paths, which reflect off the ionisphere, and "ground wave" paths, which exploit coupling of the radiowaves with the conducting ocean water to achieve extended ranges. For HF radars, instruments that operate using sky wave transmissions are often referred to as over-thehorizon (OTH) radars (e.g.. Georges, 1980), although HF ground wave radars, which are the major focus of this issue, also achieve beyond-thehorizon ranges.

Reflection (or backscatter) of electromagnetic energy from the sea surface can be expected to produce an energy "spectrum" at the receiver, even if the energy source was single-frequency, because of the complicated shape and motion of the sea surface. Interpreting these spectral returns for various transmit frequencies is the key to extracting information about the ocean. Many instruments rely on a resonant backscatter phenomenon known as "Bragg scattering," which results from coherent reflection of the transmitted energy by ocean surface waves whose wavelength is exactly one-half as long as the transmitted radar waves. The inset in Figure 2 attempts to illustrate this process by showing how energy reflected at one wave crest is precisely in phase with other energy that traveled $\frac{1}{2}$ wavelength down and $\frac{1}{2}$ wavelength back to reflect from the next wave crest. These coherent reflections result in a strong peak in the backscatter spectrum. Scatterometers exploit Bragg scattering from capillary waves $(\sim 1 \mathrm{~cm})$ to obtain information about winds. HF radars, on the other hand, exploit Bragg scattering from surface 
gravity waves $(\sim 10 \mathrm{~m})$ to obtain information about currents (and winds).

\section{Measuring Currents}

The history of HF backscatter measurements is better outlined by Teague et al. (1997). We point to the work of Crombie (1955) as the first to identify strong sea echoes in the HF band with resonant Bragg scattering. Bragg waves in the HF band happen to be "short" surface gravity waves, which can be assumed to be traveling as deepwater waves, except in very shallow depths of a few meters or less. This is important because it allows information contained in the Doppler shift of Bragg peaks to be used to estimate ocean currents.

Figure 3 illustrates the Doppler technique for ocean current determination from HF radar backscatter. It shows an actual spectrum from the Ocean Surface Current Radar (OSCR) system. The spectrum contains obvious Bragg peaks due to the presence of Bragg waves traveling toward and away from the receiver. The frequencies of these peaks are offset from that of the transmitted energy for the two following reasons: 1) the Bragg waves are moving with the deep-water phase speed given by $c=\sqrt{ }(g N / 4 \pi)$, where $\lambda$ is the wave length of the transmitted energy and $g$ is the gravitational acceleration and 2) the Bragg waves are moved by the underlying ocean current. Because the expected Doppler shift due to the Bragg waves is known, any additional Doppler shift is attributed to the current as shown in Figure 3.

It is important to keep in mind the following points about HF radar-derived currents: 1) a single radar site is capable of detecting only the component of flow traveling toward or away from the site for a given look angle; 2) the effective depth of the measurement depends on the depth of influence of the Bragg waves and is quite shallow $(\sim 1$ $\mathrm{m})$; 3) stable estimates require scattering from hundreds of wave crests plus ensemble averaging of the spectral returns, which sets the space-time resolution of the instruments; 4) the precision is limited by the frequency resolution of the Doppler spectrum and is typically $2-5 \mathrm{~cm} \mathrm{~s}^{-1}$; and 5) the accuracy is controlled by numerous factors, such as signal-to-noise ratios, pointing errors, and geometry.

Because a single radar station measures only the component of flow along a radial beam emanating from the site, "radial" currents from two or more sites should be combined to form vector surface current estimates. Figure 4 illustrates this principle using radial data from two radar sites. It also illustrates the "baseline problem" that occurs where both radar sites measure the same (or nearly the same) component of velocity, such as along the baseline between the sites or at great distances from both sites. Generally two radials must have an angle $>30^{\circ}$ and $<150^{\circ}$ to resolve the current vector. This geometric sensitivity is similar to the familiar geo-

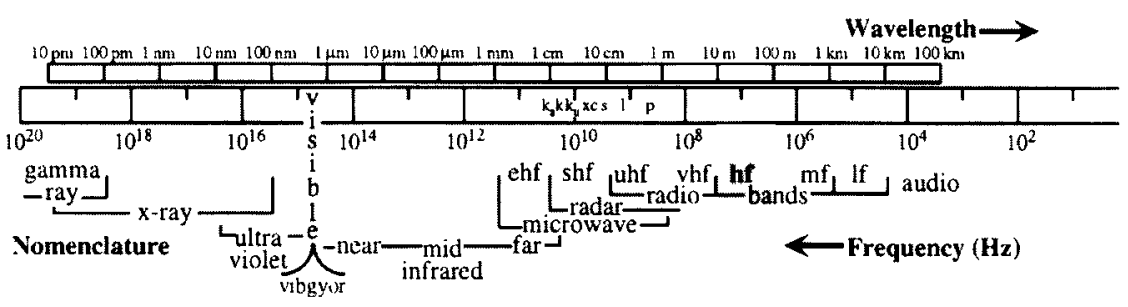

Fig. 1: Electromagnetic spectrum showing the HF band relative to other radio wave bands and the broader spectrum.

metric dilution of precision (GDOP) in the global positioning system (Chapman and Graber, 1997). If currents are assumed to be constant over several radial bins, it is also possible to estimate velocities using a single radar site as was done by Bjorkstedt and Roughgarden (1997), although the GDOP-related errors will be relatively large in this case.

The current measurement by HF radars is close to a "true" surface current measurement. Because radar pulses scatter off ocean waves, the derived currents represent an integral over a depth that is proportional to the radar wavelength. Stewart and Joy (1974) show this depth to be $\sim d=\lambda / 8 \pi$. Because wavelength depends on the radar frequency, it is feasible to use multifrequency HF radars to estimate vertical shear in the top two meters of the ocean.

Present system and coverage capabilities of HF radars are quite impressive. Measurements can be made in range as short as $1 \mathrm{~km}$ and as long as 150 $\mathrm{km}$ from the shore at a resolution of $\sim 0.3-3 \mathrm{~km}$ along a radial beam. Radio interference or high sea states can limit the actual range at times as well as

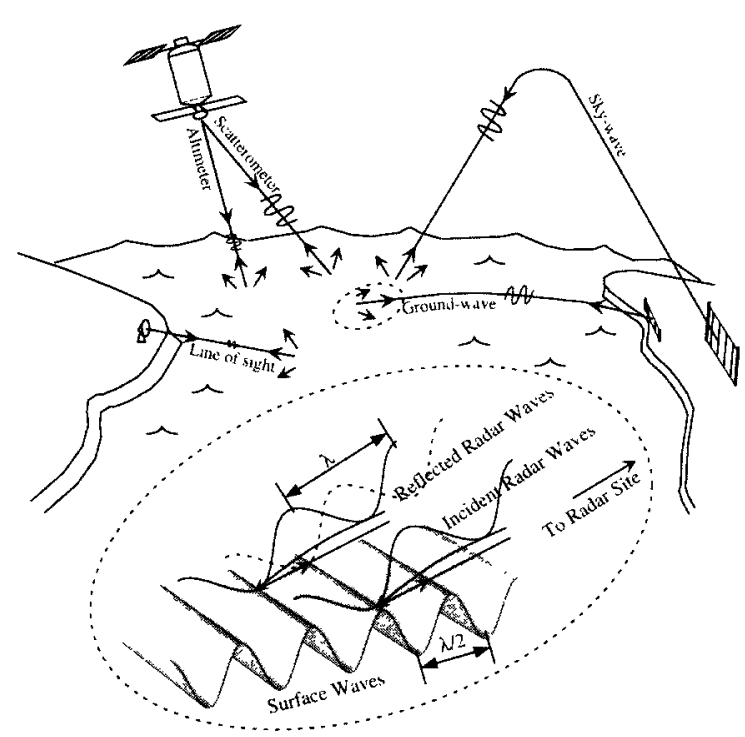

Fig. 2: Schematic representation of various remote sensing methods exploiting signals backscattered from the sea surface (adapted from Shearman, 1981). The inset illustrates the resonant Bragg scattering process that occurs due to reflection from waves whose wavelength is $\frac{1}{2}$ as long as that of the incident energy.
The current mea-

surement by HF

radars is close to a

"true" surface current

measurement. 
the ground conditions in the vicinity of the receive antennas. Wet and moist sandy soils enhance the ground wave propagation, whereas dry and rocky grounds reduce signal strengths. Typical azimuthal resolutions are $\sim 5^{\circ}$. Near the coast, this gives a measurement width of $\sim 0.5 \mathrm{~km}$; the width is $\sim 10.0$ $\mathrm{km}$ at range cells $100 \mathrm{~km}$ offshore (Fig. 4).

\section{Measuring Winds and Waves}

Although the focus of this special issue, and many of the experiments using ground wave $\mathrm{HF}$ radar systems, is on surface currents, it is also possible to extract information about surface waves and winds from HF backscatter spectra. Wave techniques are discussed by Wyatt (1997) and by Graber and Heron (1997), whereas the method for extracting wind direction is discussed by Fernandez et al. (1997). Very crudely, wave information is obtained by fitting a model of surface wave backscatter to the observed second-order portion of the spectrum (Fig. 3). That portion is due to reflections from waves at all frequencies and not just the resonant Bragg waves. Wind direction, on the other hand, is related to the ratio of the strength of the advancing and receding Bragg peaks.

\section{System Configurations}

Although the basic scattering principle is the same for all existing HF radars, distinct differences are found in the antenna configurations that transmit and receive the electromagnetic signals. The compact antenna system utilized by the Coastal Ocean Dynamics Applications Radar (CODAR) consists of crossed loops and a whip for receiving and a whip for transmitting radio pulses (Barrick $e t$ al., 1977). This antenna system is small and lends itself for deployment in highly populated and rocky coastal areas (e.g., cover photos). Radars of this type have been in use in the Monterey Bay area (Paduan and Rosenfeld, 1996; Paduan and Cook, 1997) and, with modifications, in Germany (Essen et al., 1981). The omnidirectional characteristic of the cross-loop whip combination makes it possible to scan wider ocean sectors (e.g., Fig. 4), but this requires direction-finding techniques to determine angle for a given range cell (Lipa and Barrick, 1983; Barrick and Lipa, 1997).

In contrast, linear phased-array antennas consist of numerous (typically 8-16) elements separated by one ocean wavelength and aligned normal to the principal receive direction (e.g., cover photos). These radars, such as the University of Miami's OSCR system, are positioned at the seaward edge of a beach or cliff and require open space up to $100 \mathrm{~m}$ in length. The radio pulses are transmitted from a separate antenna array, which in the case of OSCR is a four-element Yagi array. Azimuthal resolution (direction) is obtained from well-established beam forming techniques. Other radars utilizing phased arrays are found in Germany (Gurgel

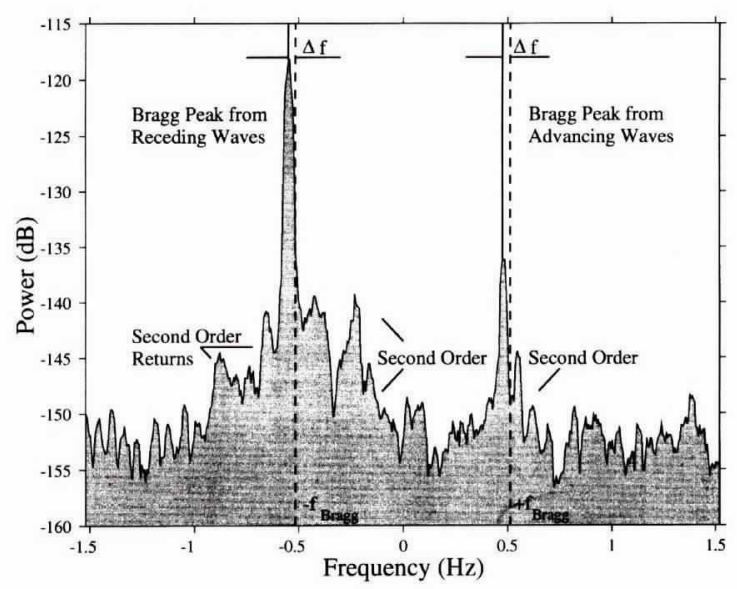

Fig. 3: Sample backscatter spectrum showing prominent Bragg peaks due to waves advancing toward and receding from the receiver. The smaller Dopper shift, $\Delta f$, is due to ocean currents that, in this example, are moving away from the receiver.

and Antonischki, 1997), Japan (Hisaki, 1996), Australia (Heron et al., 1985), France (Forget et al., 1981), Canada (Howell and Walsh, 1993), and United Kingdom (Wyatt, 1986; Prandle, 1991).

It is misleading to attempt to describe one HF radar configuration that will be optimum for all situations. Direction-finding (DF) and phasedarray systems each have their advantages and disadvantages. For example, DF systems like CODAR were developed to be able to deploy the antennas on a small coastal outcrop, or even on a building, where a long secure stretch of beach or cliff may not be available. In addition, the angular coverage from DF techniques is much greater than the, at most, $90^{\circ}$ sector that is available using phasedarray pointing techniques.

At the same time, phased-array systems have important advantages over DF systems. Because the "beam" can be steered to a particular look direc-

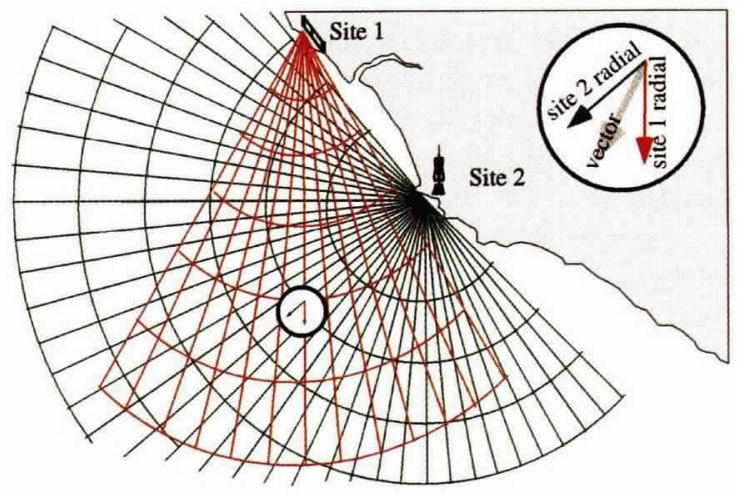

Fig. 4: Sample radial current coverage for a phasedarray radar (site 1) covering a $60^{\circ}$ swath and a direction-finding radar (site 2), which in principle can cover up to $360^{\circ}$. At overlapping ocean bins (e.g., O) a vector current estimate can be made, providing the angular separation between the radial currents is large enough. 
tion, it is possible to collect backscatter spectra from a single patch of ocean (e.g., Fig. 3) and, thereby, infer surface wave characteristics from the second-order portions of the spectra. (DF systems, by contrast, collect spectra on the basis of ocean backscatter over an entire range cell, which obscures the wave information.) The determination of wind direction is also more straightforward when using individual spectra from phased-array systems.

\section{Conclusions}

The purpose of this special issue on HF radars is to describe in simple terms how the radars work and demonstrate the usefulness and capabilities of such instrument technology for today's problems in ocean research. The following short feature articles present a wide variety of applications that are important in physical and biological oceanography in the coastal zone. Beyond their utility to the scientist, these measurements are also of great interest to both military and civilian coastal engineers, public safety officers, and planners who must maintain navigational seaways, mitigate ocean pollution, conduct search and rescue operations, and attempt to balance the health of coastal habitats, public access, and private property rights.

The advantages of HF radar as a noninvasive measurement tool that can acquire vector surface current, wave, and wind information should be obvious. However, although the concept of this technology is old, its acceptance in science, government, and industry has been slow. Today there is no reason not to develop better hardware and software components while, simultaneously, exploiting what existing systems can tell us about the ocean. By analogy, the acoustic Doppler current meter (ADCP) was, a few years ago, considered experimental and mysterious by many in the oceanographic community, whereas now its use is common. We are confident that the use of HF radars will also become commonplace and, as a result, a new level of understanding of the coastal ocean will be possible.

\section{Acknowledgments}

We thank D. Trizna, F. Herr, T. Kinder, and S. Sandgathe at ONR and C.L. Vincent at USAE/ ONR for their long-range visions of HF radar's potential. The editors of this special issue on HighFrequency Radar Remote Sensing gratefully acknowledge the continued support of ONR through grants N00014-91-J-1775 (HIRES), 92-J-1807 (REINAS), 94-1-1016 (DUCK94), 95-3-0022 (MRY BAY), 96-1-1065 (COPE), and 97-1-0348 (SHOALING WAVES).

\section{References}

Barrick, D.E., M.W. Evans and B.L. Weber, 1977: Ocean surface currents mapped by radar. Science, 198, 138-144.

Barrick. D.E. and B.J. Lipa. 1997: Evolution of bearing determination in HF current mapping radars. Oceanography, 10, 72-75.

Bjorkstedt. E.P. and J. Roughgarden. 1997: Larval transport and coastal upwelling: an application of HF radar in ecological research. Oceanography, 10, 64-67.

Chapman, R.D. and H.C. Graber, 1997: Validation of HF radar measurements. Oceanography, 10, 76-79.

Crombic. D.D. 1955: Doppler spectrum of sea echo at 13.56 Mc/s. Nature, 175, 681-682.

Essen. H.H., E. Mittelstaedt and F. Schirmer, 1981: On nearshore surface current measurements by means of radar. Dtsch. Hydrogr. Z., 34, 1-14.

Fernandez. D.M.. H.C. Graber. J.D. Paduan and D.E. Barrick, 1997: Mapping wind direction with HF radar. Oceanography, 10. 93-95.

Forget, P., P. Broche, J.C. De Maistre and A. Fontanel, 1981: Sea state frequency features observed by ground wave HF Doppler radar. Radio Sci. 16. 917-925.

Georges, T.M., 1980: Progress toward a practical skywave sea-state radar. IEEE Trans. Antennas Propag. AP-28, 751-761.

Graber. H.C. and M.L. Heron, 1997: Wave height measurements from HF radar. Oceanography, 10, 90-92.

Gurgel, K.-W. and G. Antonischki, 1997: Measurement of surface current fields with high spatial resolution by the HF radar WERA. Proceedings. IEEE Int. Geoscience and Remote Sens. Symposium. Singapore, 1820-1822.

Heron, M.L., P.E. Dexter and B.T. McGann, 1985: Parameters of the air-sea interface by high-frequency ground-wave HF Doppler radar. Aust. J. Mar. Freshwater Res., 36, 655-670.

Hisaki. Y., 1996: Nonlinear inversion of the integral equation to estimate ocean wave spectra form HF radar. Radio Sci., 31. 25-39.

Howell, R. and J. Walsh. 1993: Measurement of ocean wave spectra using narrow beam HF radar. IEEE J. Ocean. Eng., 18, 296-305.

Lipa. B.J. and D.E. Barrick, 1983: Least-squares method for the extraction of surface currents from CODAR Crossedloop data: Application at ARSLOE. IEEE J. Ocean. Eng., 8, 226-253.

Paduan, J.D. and M.S. Cook, 1997: Mapping surface currents in Monterey Bay with CODAR-type HR radar. Oceanography, 10, 49-52.

and L.K. Rosenfeld, 1996: Remotely sensed surface currents in Monterey Bay from shore-based HF radar (CODAR). J. Geophys. Res., 10I, 20669-20686.

Prandle. D., 1991: A new view of near-shore dynamics based on observations from HF radar. Prog. Oceanogr., 27, $403-438$.

Shearman. E.D.R.. 1981: Remote sensing of ocean waves, currents and surface winds by deka metric radar. In: Remote Sensing in Meteorology, Oceanography and Hydrology. A.P. Cracknell, ed. Ellis Horwood, London. 312-335.

Stewart, R.H. and J.W. Joy, 1974: HF radar measurement of surface current. Deep-Sea Res., 2I, 1039-1049.

Teague. C.C., J.F. Vesecky and D.M. Fernandez, 1997: HF radar instruments, past to present. Oceanography, 10, 40-44.

Wyatt, L.R., 1986: The measurement of the ocean wave directional spectrum from HF radar Doppler spectra. Radio Sci., 12, 473-485.

, 1997: The ocean wave directional spectrum. Oceanography, $10,85-89$. $\square$

\section{The advantages of \\ HF radar as a nonin-}

vasive measurement

tool that can acquire

vector surface cur-

rent, wave, and wind

information should be

obvious. 